\title{
Análisis de la producción científica española en energías renovables, sostenibilidad y medio ambiente (Scopus, 2003- 2009) en el contexto mundial
}

\author{
Luz María Romo Fernández \\ Vicente P. Guerrero Bote * \\ Félix Moya Anegón **
}

Artículo recibido:

7 de noviembre de 2012.

Artículo aceptado:

18 de febrero de 2013.

\section{RESUMEN}

Este trabajo analiza la producción científica de España para el periodo 2003-2009 en el área de las Energías Renovables, Sostenibilidad (otros dicen sustentabilidad) y Medio Ambiente utilizando la base de datos Scopus. En primer lugar se muestra un estudio de contexto comparando la producción e impacto con los países más desarrollados. Y en segundo lugar se hace un estudio interno de las contribuciones de las instituciones españolas así como de las revistas utilizadas por los científicos para la publicación de los trabajos. El estudio muestra que España incrementa notablemente la producción científica, al pasar de la posición

* Los dos autores pertenecen a la Universidad de Extremadura, Scimago Group, España. (Luz: Imromfer@alcazaba.unex.es); (Vicente: guerrero@unex.es)

** CSIC, CCHS, IPP, Scimago Group, España. felix@ugr.es

INVESTIGACIÓN BIBLIOTECOLÓGICA, Vol. 27, Núm.60, mayo/agosto, 2013, México, ISSN: 0187-358X. pp. 125-151 
decimoquinta a nivel mundial a la sexta posición, y que eso no se consigue a costa de reducir el impacto sino incrementándolo. Como instituciones destacan el CIEMAT y la UPM por su producción y la U. Zaragoza y el CSIC, tanto por la producción como por el impacto.

Palabras clave: Energías Renovables; Sostenibilidad y Medio Ambiente; España; Producción Científica; Instituciones de Investigación.

\section{ABSTRACT}

Analysis of the Spanish scientific production in Renewable Energy, Sustainability and the Environment (Scopus, 2003-2009) in the global context

Luz-Maria Romo-Fernández, Vicente-P. Guerrero-Bote and Félix Moya-Anegón

Using the Scopus database for the period 2003-2009, this paper analyzes the scientific production in the area of Renewable Energy, Sustainability and Environment in Spain. Initially, researchers compare Spain's scientific production and impact against that of the leading developed countries. This comparison is followed by an examination of the contributions of Spanish researchers and the journals in which they publish, showing that Spain's scientific output rank advanced significantly from fifteenth to the sixth in the world. Moreover, the impact of Spain's research increased during this period. The study found CIEMAT and UPM are among Spain's leading producers of scientific research, while the University of Zaragoza and CSIC are highly ranked in both production and impact.

Keywords: Renewable Energy; Sustainability and the Environment; Spain; Scientific Production; Research Institutions.

\section{INTRODUCCIÓN}

Dor medio ambiente podemos entender todo lo que rodea a las personas.
En los años 70 la Comisión de la ONU para el Medio Ambiente y el De-
sarrollo determinó que el camino que había tomado la sociedad deterioraba 
el medio ambiente, a la vez que sumía en la pobreza a una mayor parte de la misma. La necesidad de energía para el desarrollo económico y social ha sido siempre una de las mayores preocupaciones; gran parte de las fuentes de energía actuales son finitas y producen un gran impacto en el medio ambiente, el cual está produciendo cambio climático. La sostenibilidad se definió por primera vez en el informe Brundtland (1987) como la satisfacción de las necesidades de la actual generación sin sacrificar la capacidad de futuras generaciones para satisfacer sus propias necesidades. Esta definición ha dado lugar a lo que Komiyama y Takeuchi (2006) han llamado Ciencia de la Sostenibilidad, que incluye una dimensión social. Las energías renovables han despertado un creciente interés porque se obtienen de la naturaleza, porque son prácticamente inagotables, bien por el volumen de la fuente o por su renovación por procesos naturales y porque son seguras y limpias (Simon et al., 2010).

El traslado a los gobiernos de este interés social se ha materializado en una legislación más exigente, que ha supuesto que las empresas privadas están asumiendo este interés, y apoyando tanto la explotación de las mismas, mediante ayudas que igualen su rentabilidad frente a las convencionales, como otras ayudas y programas que fomenten la investigación y el desarrollo tecnológico del sector.

Dentro del departamento de Energía de EE.UU. está la EERE (Office of Energy Efficiency and Renewable Energy) que fomenta la investigación y el desarrollo tanto en eficiencia energética como en energías renovables. En la Unión Europea (UE), el séptimo programa marco incluye un campo temático sobre Energía (dedicado a la financiación de energías renovables en su mayor parte) y otro sobre Medio Ambiente (incluye el cambio climático y financia, entre otras cosas, estudios de sostenibilidad del medio ambiente tanto natural como urbano). A nivel español, dentro del VI Plan Nacional de Investigación Científica, Desarrollo e Innovación Tecnológica, tenemos una acción estratégica de Energía y Cambio Climático.

A pesar del auge de los estudios bibliométricos y del creciente interés en Energías Renovables, Sostenibilidad y Medio Ambiente, nos encontramos con un gran vacío en cuanto a análisis de dominio científico en dicha disciplina, seguramente por ser un campo tan reciente.

Existen algunos estudios cienciométricos que analizan este tema utilizando técnicas bibliométricas (Thomas, 1992; Uzun, 2002; Hassan, 2005; Tsay, 2008; Kajikawa et al. 2008; Kajikawa y Takeda, 2008; Celiktas et al., 2009; Romo Fernández, et al., 2011 y 2012; Sanz-Casado et al., 2012; Dong et al., 2012) y que han permitido observar el progreso de la ciencia y la tecnología, y han sido utilizados para satisfacer la necesidad de tener una visión global sobre las actividades de investigación. 
Thomas (1992) evalúa el trabajo de los grupos de investigación que trabajan en el campo de la biomasa en las áreas externas a EE.UU. y la UE El autor consideró dos elementos claves: la medida de la productividad científica y el estudio de los factores que afectan al funcionamiento de la investigación.

Seguidamente, con un importante salto en el tiempo, Uzun (2002) comparó los resultados de la investigación y las prioridades de 25 países en energías renovables para los periodos 1996-1997 y 1998-1999, tomando como medidas el número de publicaciones, su incremento y el índice de prioridades de investigación.

Hassan (2005), reconociendo el papel que juega la ciencia y la tecnología en el desarrollo de las pilas de combustible, trata de caracterizar la evolución de la estructura de estas pilas en la década de 1990, utilizando datos sobre patentes y publicaciones científicas.

Tsay (2008) explora las características de la literatura de la energía del hidrógeno desde 1965 a 2005 utilizando Science Citation Index Expanded. Los resultados de este trabajo revelan que la literatura sobre la energía del hidrógeno ha crecido de forma exponencial con una tasa de crecimiento anual de alrededor del $18 \%$ en la última década.

Kajikawa et al. (2008) hicieron un análisis de la red de citas de las publicaciones científicas sobre energías renovables para conocer la estructura actual de la investigación en este dominio. Los resultados confirman que dentro de las investigaciones relacionadas con este campo, aquellas correspondientes a las células de combustible (fuel) y a las células solares son las que están experimentando un crecimiento más rápido.

Kajikawa y Takeda (2008) analizaron las subáreas de biomasa y biocarburantes, que han ganado un creciente interés como energía sostenible. En su artículo también realizan un análisis de la red de citas de las publicaciones científicas utilizando técnicas de arracimamiento (clustering). Según los resultados, dentro de la investigación en biomasa y biocarburantes, las correspondientes a la producción de biocombustible (biofuel) e hidrógeno se desarrollan más rápidamente.

Celiktas et al. (2009) intentaron descubrir las tendencias de la investigación en energías renovables durante un amplio periodo (1980-2008) pero se centraron únicamente en Turquía. Los autores concluyen que las publicaciones sobre biomasa y sistemas de conversión, así como sobre sistemas de energía solar, fueron las predominantes. También señalan que tanto el número de publicaciones como el de citas se incrementaron en la última década, y más de la mitad del total de documentos fueron publicados en los últimos cuatro años.

Romo Fernández et al. (2011) proporcionan una visión general de la investigación de los principales países europeos en el área de Energía Renovable 
durante el periodo 2002-2007, utilizando la base datos de literatura científica Scopus (Elsevier). Los resultados muestran que la producción mundial y europea se duplicó en el periodo estudiado y que Europa, que llegó a tener 40 \% de la producción mundial, creció a menor ritmo que el resto del mundo.

Y por último, en un trabajo posterior Romo Fernández et al. (2012) muestran la producción científica del mundo tanto por países e instituciones de investigación como por revistas científicas para el periodo 2003-2008. Los resultados indican que la producción total del mundo aumenta a lo largo del periodo estudiado y que con el paso de los años este tema está adquiriendo un gran interés en el ámbito científico.

Nuestro estudio pretende analizar la producción científica del área temática específica "Renewable Energy, Sustainability and the Environment" incluida en la base de datos Scopus, utilizando indicadores cienciométricos para el periodo 2003-2009, con el fin de conocer la evolución de la investigación en dicha área en el caso de España.

\section{MATERIAL Y MÉTODOS}

En este trabajo nos hemos basado en los outputs, es decir, los resultados producidos, centrándonos exclusivamente en los trabajos científicos publicados, ya que constituyen el principal producto de la labor científica. Dentro de dicha producción científica nos hemos centrado en la producción primaria, es decir, los tipos documentales correspondientes a Artículos, Revisiones y Contribuciones a Congresos, que son los que suponen realmente aportaciones científicas y los que reciben la mayor parte de las citas.

Como es ampliamente conocido no todas las publicaciones tienen el mismo valor para los científicos. Si bien es difícil establecer una clara diferencia entre las publicaciones de un cierto nivel y las que no lo tienen, está ampliamente aceptado considerar las que se encuentran en las grandes bases de datos bibliográficas (Web of Science y Scopus principalmente) como las más importantes en cada área temática.

Desde noviembre de 2004 (Hane, 2004) está disponible la base de datos bibliográfica multidisciplinaria Scopus, creada por Elsevier. Con casi una década de este producto en el mercado, ya existen varios trabajos que intentan caracterizar y analizar dicha base de datos (Jacso, 2004; Laguardia, 2005; Codina, 2005; Archambault et al., 2009; Moya-Anegón et al., 2007; Leydesdorff, Moya-Anegón, Guerrero-Bote, 2010).

En Scopus, los documentos están clasificados por Áreas Temáticas (Subject Area) y por Áreas Temáticas Específicas (Specific Subject Areas). Hay 295 
Áreas Temáticas Específicas que están agrupadas en 26 Áreas Temáticas. Además, está el Área Temática general que contiene revistas multidisciplinares como Nature o Science. Las Áreas Temáticas están agrupadas en cuatro Categorías en la página "Basic Search" (www.scopus.com visitado el 8 de octubre de 2011).

Las cuatro Categorías son (mantenemos los títulos de las Categorías y Áreas Temáticas en inglés):

- Life Sciences (3950 revistas): Agricultural \& Biological Sciences; Biochemistry, Genetics \& Molecular Biology; Immunology \& Microbiology; Neuroscience; Pharmacology, Toxicology \& Pharmaceutics.

- Physical Sciences (6 350 revistas): Chemical Engineering; Chemistry; Computer Science; Earth \& Planetary Science; Energy; Engineering; Environmental Science; Materials Science; Mathematics; Physics \& Astronomy.

- Social Sciences (5 900 revistas): Arts \& Humanities; Business, Management \& Accounting; Decision Sciences; Economics, Econometrics and Finance; Psychology; Social Sciences.

- Health Sciences (6 200 revistas): Medicine; Nursing; Veterinary; Dentistry; Health Professions.

Dentro de la categoría correspondiente a las Ciencias Físicas (Pbysical Sciences) está el Área Temática correspondiente a Energía (Energy), que se compone de cinco Áreas Temáticas Específicas, una de las cuales es la correspondiente a Energías Renovables, Sostenibilidad y Medio Ambiente (Renewable Energy, Sustainability and the Environment). Dicha Área Temática Específica, como su nombre lo indica, incluye trabajos dedicados a las energías renovables, a sostenibilidad y a su relación con el medio ambiente.

En nuestro trabajo hemos utilizado Scimago Institutions Rankings (SIR) (http://www.scimagoir.com/), una herramienta para la evaluación de las universidades e instituciones de todo el mundo que priorizan la investigación. La jerarquía (ranking) muestra las actividades de investigación de instituciones de todo el mundo que destacan en producción científica, incluidas las universidades, agencias gubernamentales, laboratorios de investigación, hospitales y muchos más. Con ese fin se han normalizado los nombres de las instituciones que aparecen en los campos de filiación.

Para este trabajo se han utilizado los artículos, revisiones y contribuciones a congresos clasificados en el área temática especializada correspondiente a Energías Renovables, Sostenibilidad y Medio Ambiente publicados en el periodo 2003-2009. Los datos fueron descargados en diciembre de 2011. 
El SJR (Scimago Journal Rank) es un indicador desarrollado por el grupo de investigación SCImago ${ }^{1}$ que muestra la visibilidad de las revistas contenidas en Scopus desde 1996 (González-Pereira, Guerrero-Bote, Moya-Anegón et al., 2010; Bollen et al., 2009; Guerrero-Bote y Moya-Anegón, 2012). Está basado en la transferencia de prestigio o influencia desde una revista a otra a través de las referencias. Es un indicador de las revistas independiente de su tamaño, que pondera las citas recibidas por las revistas en una ventana de tres años con el prestigio de la revista citante.

La Citación Normalizada de cada trabajo se ha calculado dividiendo su citación por el promedio de citas mundial de los documentos de la misma Área Temática Específica, año y tipo documental. Para calcular la citación normalizada de la producción de un dominio se calcula el promedio para todos los documentos del dominio. La citación normalizada mundial será siempre 1, una citación normalizada de 1.3 significará que se supera en un $30 \%$ el promedio mundial, mientras que un valor de 0.85 significará que es un $15 \%$ menor que el promedio mundial.

La Tasa de Variación (TV) utilizada es la diferencia porcentual del número de trabajos en el año 2009 en relación con el total de la producción del año 2003.

El indicador IET (Índice de Especialización Temática) (Moya-Anegón et al., 2004) refleja el nivel de especialización, entendido como el esfuerzo relativo que una comunidad o agente le dedica a una disciplina o área temática. Cuantifica de forma relativa el número de documentos producidos en una disciplina concreta por un determinado colectivo con respecto a otro colectivo:

$$
I E T_{C E / M}=\frac{\frac{N \operatorname{docc}_{C E}}{N \operatorname{docc}_{E}}}{\frac{N \operatorname{docc}_{C M}}{N \operatorname{docc}_{M}}}=\frac{\% \operatorname{docc}_{C E}}{\% \operatorname{docc}_{C M}}
$$

Donde:

- $N d o c c_{C E}$ es el número de documentos del colectivo $E$ en el campo $C$ (análogamente $\mathrm{Ndocc}_{C M}$ para el colectivo de referencia, en nuestro caso la producción mundial)

- $N d o c c_{E}$ es el número total de documentos del colectivo $E$ (análogamente $\mathrm{N} d o c c_{M}$ ) 
- \% Ndocc $C E$ es el porcentaje que suponen, dentro del total de documentos primarios del colectivo $E$, los del campo temático $C$ (de manera análoga se define $\% \mathrm{Ndocc}_{C M}$ ).

Por último, el indicador Tasa de Colaboración Internacional representa el porcentaje de documentos firmados en colaboración con autores de otros países respecto del total de documentos del dominio o agregado.

\section{Resultados}

Comenzamos nuestro análisis con un pequeño estudio de contexto comparado con la producción mundial así como con los países más destacados en el área de estudio.

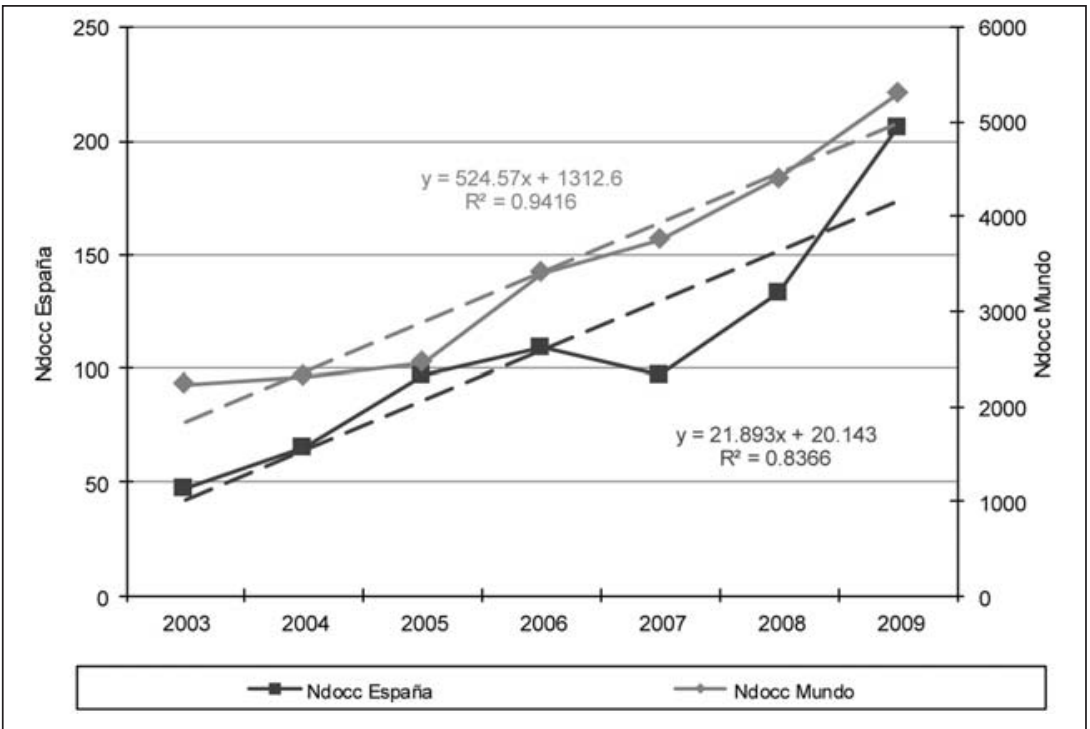

Figura 1. Evolución temporal de la producción primaria de España y el mundo (periodo 2003-2009)

Como se puede observar en la Figura 1, la producción primaria de España aumentó notablemente a lo largo del periodo estudiado, llegando a cuadruplicarse en estos seis años. También creció mucho la producción primaria mundial, sin embargo, mientras que la tasa de variación de la producción primaria española fue de $338 \%$, la mundial fue de $137 \%$. La correlación lineal de ambas producciones con los años es significativa estadísticamente (al nivel de significación $\alpha=0.0039$ en el caso de la producción española y al nivel de 
$\alpha=0.00029$ en el caso de la producción mundial) y por tanto son aceptables los ajustes lineales. Los datos mostrados aquí son similares a los mostrados en otros trabajos (Romo-Fernández et al., 2011 y 2012).

España contó con una producción alta y creciente en el periodo excepto en el año 2007 que sufrió un pequeño descenso.

Como podemos observar en la Tabla I (página siguiente), los países que contaron con una producción superior a 1000 documentos fueron Estados Unidos, China, Reino Unido, India, Turquía y Japón.

En cuanto a Tasa de Variación se refiere podemos decir que China fue el país que mayor crecimiento experimentó en el año 2009 con respecto a 2003, con un 507.14 \%, seguido de España. Sin embargo, mientras en China esta tasa de variación de la producción científica en el área de estudio no llegó a duplicar la tasa de variación de la producción científica total, en España casi la quintuplicó (fuente: http://www.scimagojr.com/ [consultado el 12 de octubre de 2012]). Esto sitúa a España en la novena posición por producción igual que el estudio de Dong et al. (2012), aunque el mismo se refiere exclusivamente a Energía Solar y a un periodo ligeramente diferente. Sin embargo, la posición de España no fue estática sino que ascendió durante el periodo de la posición décimo quinta a la sexta posición, como también se muestra en el reciente trabajo de Sanz-Casado et al. (2012).

Los países que registraron mayor esfuerzo/especialización en esta área del Índice de Especialización Temática (IET) respecto al mundo en cuanto a energía renovable, sostenibilidad y medio ambiente fueron Turquía y Grecia. En este sentido, España ha hecho un esfuerzo/especialización en este área superior al promedio mundial, aunque hay seis países por delante, de los cuales sólo dos tuvieron una mayor producción.

España se posicionó en séptimo lugar en citación normalizada superando el promedio de citación mundial en un 15 \% en todo el periodo. Llegó a estar en 2004 un $3 \%$ por debajo del promedio mundial, pero en 2009 superó en $23 \%$ dicho promedio. Se situó en cuarta posición en cuanto a países que presentaban un mayor crecimiento anual medio en este indicador, junto con Dinamarca, Japón, Francia y Estados Unidos. De hecho si analizamos la serie temporal se observa que España pasó de la undécima posición en 2003 a la séptima en 2009. Esto supone que el gran incremento de producción durante el periodo no supuso una merma del impacto, sino que también aumentó.

Si analizamos manualmente los valores medios de las variables utilizadas para los países, podríamos establecer los siguientes grupos:

- Grupo 1 (sobresaliente): formado por países con altos valores en producción, y valores medios/altos tanto en índice de especialización temática 


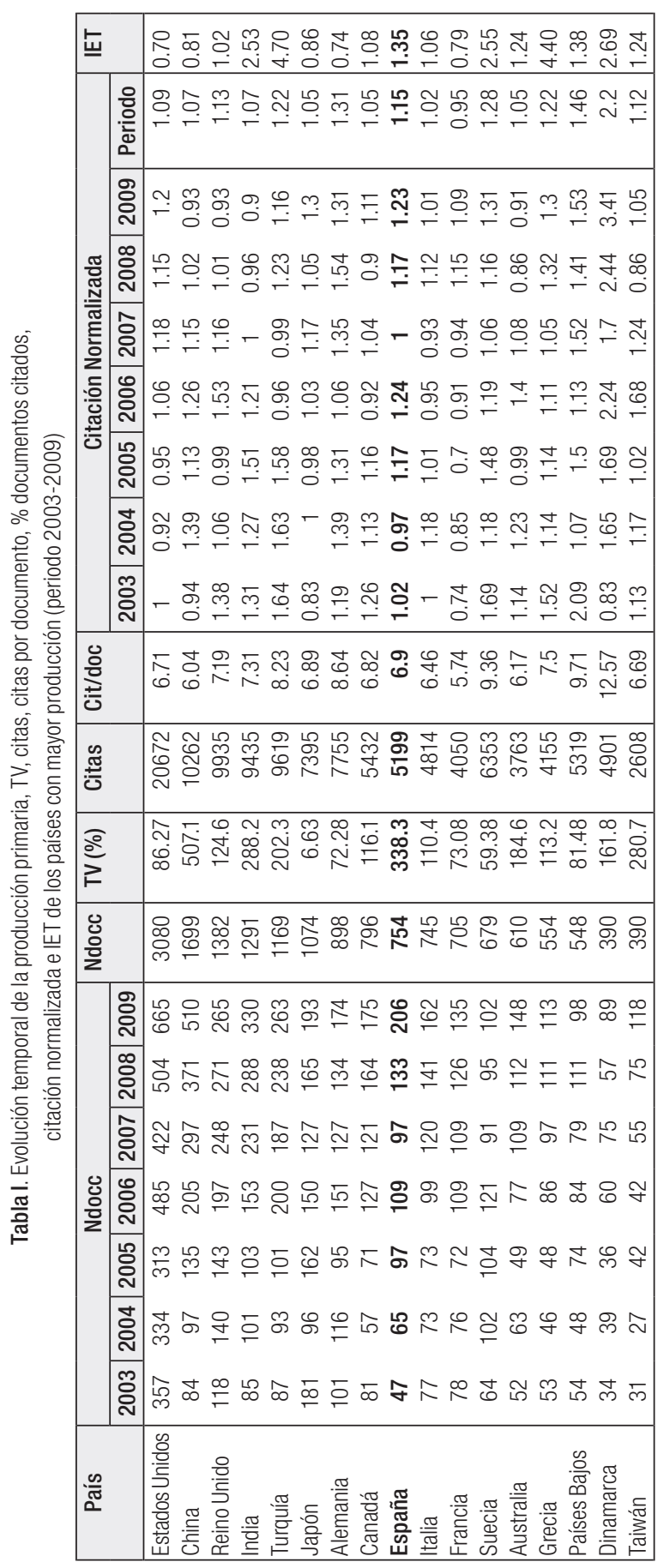


como en citación normalizada. Podemos decir que el prototipo de este grupo es Turquía; además, incluimos a Reino Unido, India, Canadá, Italia y España.

- Grupo 2 (países avanzados): caracterizado por contar con países con altos valores en producción y citación normalizada, pero en este caso con baja especialización. Estados Unidos podría ser el prototipo. Además, otros países encuadrados aquí serían: China, Japón, Alemania y Francia.

- Grupo 3 (especializado): formado por aquellos países que contaban con una producción media y valores medios/altos en citación normalizada e índice de especialización. El prototipo sería Grecia. Además incluimos en este grupo a Suecia, Australia, Países Bajos, Dinamarca y Taiwán.

En la Figura 2 (página siguiente) podemos observar el IET (eje horizontal) y la citación normalizada (eje vertical) de los países que tuvieron mayor producción en el campo de las Energías Renovables en el periodo estudiado. El volumen del círculo es proporcional a la producción primaria en el área en cada país. Si dividimos la gráfica en cuatro cuadrantes, tomando como centro la posición de España, podemos ver que en el cuadrante superior derecho, mayor impacto (citación normalizada) y especialización (IET) que España, están Dinamarca, Suecia, Grecia, Países Bajos (con una especialización muy similar a la de España) y Turquía, de los cuales solamente el último tuvo una mayor producción primaria que España. En el cuadrante superior izquierdo, es decir, con mayor impacto y menor especialización que España, solamente está Alemania. En el cuadrante inferior derecho, que indica mayor especialización pero menor impacto que España solamente está India. El resto de países tienen tanto menor esfuerzo como impacto que España. Estos resultados son similares a los mostrados en estudios previos (RomoFernández et al., 2011 y 2012).

En la Tabla II (p. 137) se muestra la colaboración internacional de España con el resto de países del mundo en el periodo 2003-2009. Además de los documentos en colaboración y la citación que éstos reciben podemos ver los porcentajes que supusieron, tanto el número de documentos como la citación, respecto a la producción española. Si nos fijamos, salvo el caso de los tres países finales, el porcentaje de citación fue mayor que el porcentaje de documentos, lo que indica una mayor citación de los documentos en colaboración, como se ha mostrado en los estudios sobre la colaboración internacional (Lancho-Barrantes et al., 2012; Guerrero-Bote et al., 2013). 


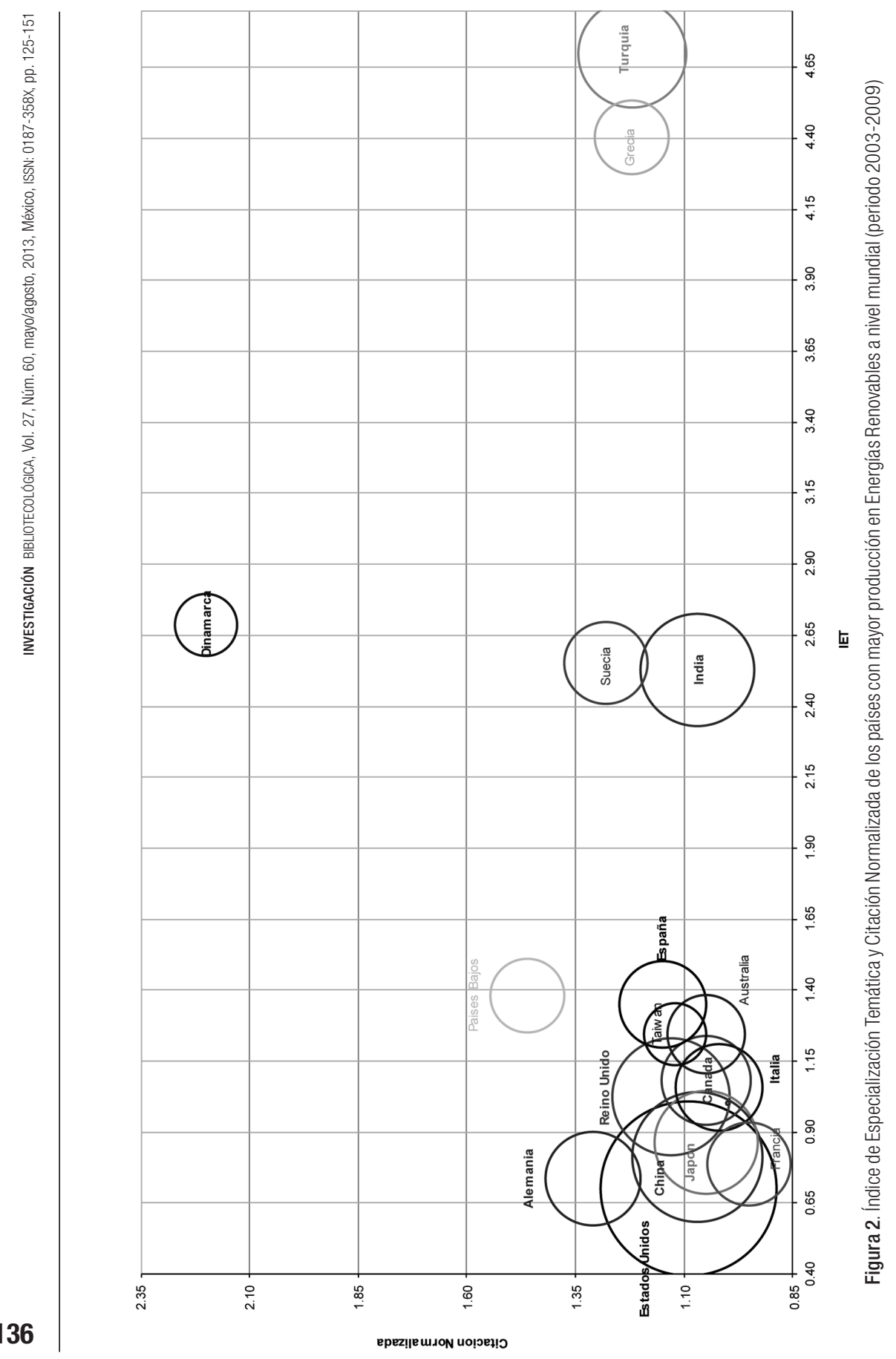


Tabla II. Colaboración internacional de España con el mundo (periodo 2003-2009). Ordenado por el número de documentos

\begin{tabular}{|l|l|c|c|c|c|}
\hline \multicolumn{1}{|c|}{ País } & Abrev. & Doc & Citas & $\%$ Doc & \% Cit \\
\hline Alemania & DEU & 44 & 421 & 5.84 & 8.10 \\
\hline Reino Unido & GBR & 31 & 330 & 4.11 & 6.35 \\
\hline Estados Unidos & USA & 23 & 168 & 3.05 & 3.23 \\
\hline Italia & ITA & 22 & 234 & 2.92 & 4.50 \\
\hline Francia & FRA & 20 & 188 & 2.65 & 3.62 \\
\hline Países Bajos & NLD & 19 & 198 & 2.52 & 3.81 \\
\hline Dinamarca & DNK & 14 & 189 & 1.86 & 3.64 \\
\hline Grecia & GRC & 11 & 123 & 1.46 & 2.37 \\
\hline Suecia & SWE & 10 & 203 & 1.33 & 3.90 \\
\hline Canadá & CAN & 5 & 68 & 0.66 & 1.31 \\
\hline Japón & JPN & 4 & 53 & 0.53 & 1.02 \\
\hline Australia & AUS & 3 & 31 & 0.40 & 0.60 \\
\hline Turquía & TUR & 3 & 6 & 0.40 & 0.12 \\
\hline China & CHN & 2 & 9 & 0.27 & 0.17 \\
\hline India & IND & 1 & 0 & 0.13 & 0 \\
\hline
\end{tabular}

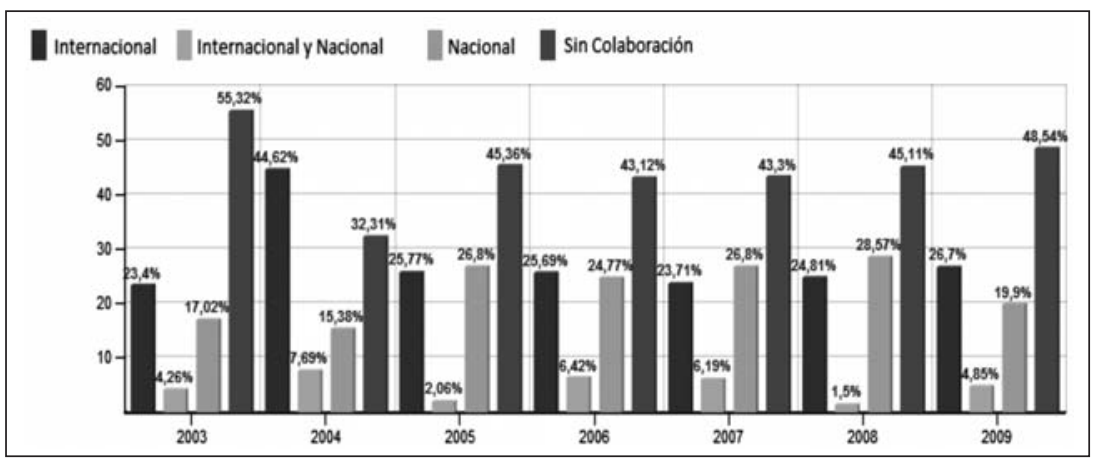

Figura 3. Evolución temporal de la Tasa de Colaboración de España (periodo 2003-2009)

En la Figura 3 podemos ver cómo la tasa de trabajos sin colaboración está cercana al $50 \%$ (se aleja más en 2004), siendo superior en la mayor parte de los casos a las otras tasas. La tasa de colaboración nacional fue superior a la internacional en tres años, aunque en promedio ganó la internacional. Lógicamente la tasa menor fue la de documentos con colaboración tanto nacional como internacional. En la evolución, las tasas del año 2004 fueron bastante diferentes al resto, con una alta colaboración internacional, y se intuye un ligero incremento de las tasas de colaboración, pero sin una tendencia clara. 


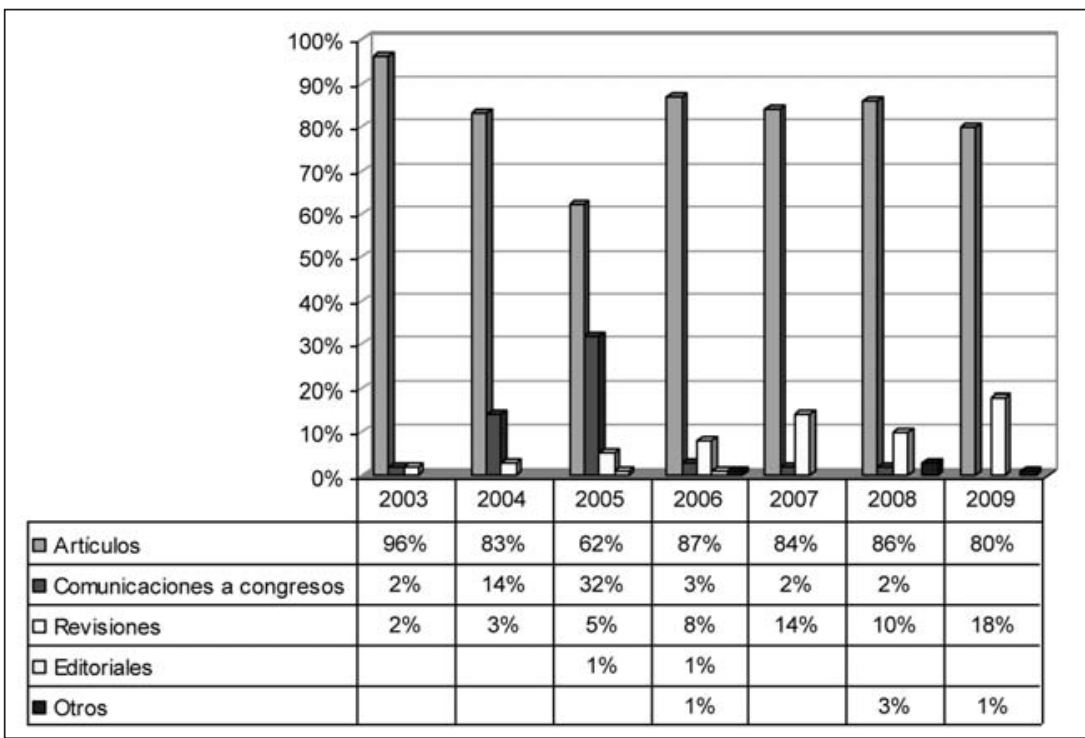

Figura 4. Porcentajes de producción según la tipología documental de la categoría Renewable Energy, Sustainability and the Environment (periodo 2003-2009)

Como podemos observar en la Figura 4, el artículo científico es la tipología documental más utilizada con diferencia del resto. Seguidamente encontramos las revisiones y las contribuciones a congresos. En el año 2005 se observa un mayor porcentaje de contribuciones a congresos, esto se debió principalmente al "3rd European Meeting on Solar Chemistry and Photocatalysis: Environmental Applications (SPEA-3)", celebrado en Barcelona en 2004 y al "International Conference on the Physics, Chemistry and Engineering of Solar Cells", celebrado en Badajoz, Spain en 2004, que publicaron una selección de contribuciones en las revistas Solar Energy y Solar Energy Material and Solar Cells.

Tabla III. Producción primaria, citas, citas/documento, citación normalizada e IET de las instituciones españolas (producción igual o superior a 8 documentos) (periodo 2003-2009).

\begin{tabular}{|l|l|c|c|c|c|c|}
\hline \multicolumn{1}{|c|}{ Institución } & ABREV & Ndocc & $\%$ TV & $\begin{array}{c}\text { Citación } \\
\text { Normalizada }\end{array}$ & \% TV CN & IET \\
\hline $\begin{array}{l}\text { Centro de Investigaciones Energéticas, } \\
\text { Medioambientales y Tecnológicas }\end{array}$ & CIEMAT & 107 & 171.4 & 1.07 & -73.7 & 26.2 \\
\hline Universidad Politécnica de Madrid & UPM & 92 & 116.7 & 0.88 & 70.2 & 6.56 \\
\hline Universidad de Zaragoza & UNIZAR & 52 & & 1.53 & & 3.61 \\
\hline $\begin{array}{l}\text { Consejo Superior de } \\
\text { Investigaciones Científicas }\end{array}$ & CSIC & 46 & 600 & 1.67 & 130.4 & 0.5 \\
\hline Universidad de Jaén & UJAEN & 40 & 350 & 1.13 & 25.7 & 9.55 \\
\hline Universitat Autónoma de Barcelona & UAB & 34 & 1200 & 1.22 & -15.9 & 1.35 \\
\hline
\end{tabular}




\begin{tabular}{|l|l|c|c|c|c|c|}
\hline Universidad Carlos III de Madrid & UC3M & 30 & 1000 & 1.07 & -77.4 & 4.08 \\
\hline Universitat Politécnica de Catalunya & UPC & 29 & 200 & 0.81 & -78.6 & 1.34 \\
\hline Universidad de Sevilla & US & 26 & 100 & 1.11 & 2162.5 & 1.69 \\
\hline Universidad de Valladolid & UVA & 26 & 50 & 1.01 & 30.9 & 3.25 \\
\hline Universidad Politécnica de Valencia & UPV & 26 & 150 & 1.23 & 119.7 & 1.7 \\
\hline Universidad del País Vasco & UPV/EHU & 25 & 400 & 1.23 & 181.4 & 1.7 \\
\hline Universidad de Almería & UAL & 19 & & 0.87 & & 5.38 \\
\hline Universidad de Granada & UGR & 18 & & 0.5 & & 1.02 \\
\hline Universidad de Vigo & UVIGO & 18 & & 0.61 & & 2.04 \\
\hline $\begin{array}{l}\text { Universidad de las Palmas de Gran } \\
\text { Canaria }\end{array}$ & ULPGC & 16 & 0 & 1.42 & 66.7 & 3.93 \\
\hline Universidad Complutense de Madrid & UCM & 15 & 133.3 & 0.74 & 80.9 & 0.56 \\
\hline Universidad de Castilla-La Mancha & UCLM & 15 & & 1.15 & & 1.78 \\
\hline Universidad de Oviedo & UNIOVI & 15 & 600 & 0.89 & 112.8 & 1.36 \\
\hline Universidad de Santiago de Compostela & USC & 15 & 800 & 1.42 & & 0.99 \\
\hline Universidad Politécnica de Cartagena & UPCT & 14 & 150 & 0.62 & 38.5 & 4.74 \\
\hline Universidad de Cádiz & UCA & 13 & & 1.71 & & 3.26 \\
\hline $\begin{array}{l}\text { Universidad Nacional de Educación a } \\
\text { Distancia }\end{array}$ & UNED & 13 & & 0.86 & & 3.5 \\
\hline Universitat de Barcelona & & & & & & \\
\hline Universidad de La Rioja & UB & 13 & & 1.31 & & 0.4 \\
\hline Universitat de Lleida & UNIRIOJA & 12 & & 1.31 & & 8.6 \\
\hline Instituto de Prospectiva Tecnológica & UDL & 11 & 100 & 1.73 & 53.7 & 3.13 \\
\hline Instituto Tecnológico de Canarias & ITC & 10 & 50 & 1.15 & 57.0 & 46.47 \\
\hline $\begin{array}{l}\text { Institut de Recerca i Tecnologia } \\
\text { Agroalimentaries Barcelona }\end{array}$ & IRTA & 9 & -50 & 1.41 & 234.4 & 163.93 \\
\hline Universidad de Córdoba & UCO & 9 & -50 & 1.59 & -19.4 & 1.46 \\
\hline Universidad de Extremadura & UEX & 9 & & 0.71 & & 1.47 \\
\hline Universidad de Huelva & UHU & 9 & & 1.57 & & 4.17 \\
\hline Universidad de Málaga & UMI & 8 & 0 & 0.69 & 134.8 & 1 \\
\hline Universitat Jaume I & & 3.09 & & 1.7 \\
\hline
\end{tabular}

Contamos con un total de 109 instituciones españolas con producción en Energía Renovable, Sostenibilidad y Medio Ambiente. En la Tabla III nos hemos centrado en aquellas 34 que contaban con una producción igual o superior a 8 documentos y, de este grupo, encontramos que 29 de ellas pertenecían al sector universitario y 5 al sector público. Las tasas de variación no se calcularon si la institución no dispuso de producción en 2003. Hay centros de investigación especializados que se dedican a campos específicos, aunque la mayoría son generalistas, e incluso muchos de ellos tienen también otras misiones como la docencia.

En cuanto a producción científica encontramos en primer lugar el Centro de Investigaciones Energéticas, Medioambientales y Tecnológicas que es la institución con mayor número de documentos en el periodo de estudio, si 
bien en crecimiento anual promedio esta institución está en la posición 9. Seguidamente encontramos la Universidad Politécnica de Madrid que en cuanto a crecimiento anual medio se situó en la posición 13. En la tercera posición en cuanto a producción científica quedó la Universidad de Zaragoza, que no tuvo producción en 2003.

La Universitat Autónoma de Barcelona, la Universidad Carlos III de Madrid, la Universidad de Santiago de Compostela y el Consejo Superior de Investigaciones Científicas fueron las instituciones que mayor crecimiento anual medio en producción experimentaron en el periodo estudiado.

Volviendo a la citación normalizada de las instituciones objeto de estudio, podemos ver que más del $60 \%$ de ellas superaron el promedio mundial ya que alcanzaron valores superiores a 1 . De las instituciones con 30 trabajos o más (las siete más productivas), llama la atención el alto impacto del CSIC (1.67) y el de la Universidad de Zaragoza (1.53).

Si hablamos del crecimiento anual medio, las primeras posiciones las ocuparon la Universidad de Sevilla, la Universidad del País Vasco y el Consejo Superior de Investigaciones Científicas (de entre las que tuvieron producción tanto en 2003 como en 2009).

Entre las instituciones más productivas que mayor esfuerzo realizan respecto al mundo en Energías Renovables, Sostenibilidad y Medio Ambiente está el Centro de Investigaciones Energéticas, Medioambientales y Tecnológicas. Se puede observar que la mayoría de las universidades son generalistas, ya que cuentan con poca especialización. Entre las universidades más productivas llama la atención el gran esfuerzo realizado por la U. de Jaén, la U. Politécnica de Madrid, la U. Carlos III y la U. de Zaragoza.

En la Figura 5 se ha representado el tamaño (número de trabajos publicados) y el impacto (citación normalizada) de la producción científica primaria de las mismas instituciones de la Tabla III.

De acuerdo con los indicadores manejados para las instituciones mencionadas se pueden establecer cuatro grupos:

- Grupo 1. Formado por las instituciones públicas de investigación distintas a las Universidades. Éstas se caracterizan por una citación normalizada superior a la unidad y una especialización alta, a excepción del CSIC. El CSIC y el CIEMAT tienen una mayor producción que el resto, conformado por el Instituto de Prospectiva Tecnológica, el Instituto Tecnológico de Canarias y el Institut de Recerca i Tecnologia Agroalimentaries Barcelona.

- Grupo 2. Compuesto por Universidades que obtuvieron una citación normalizada, más de un $30 \%$ superior al promedio mundial. A excepción 


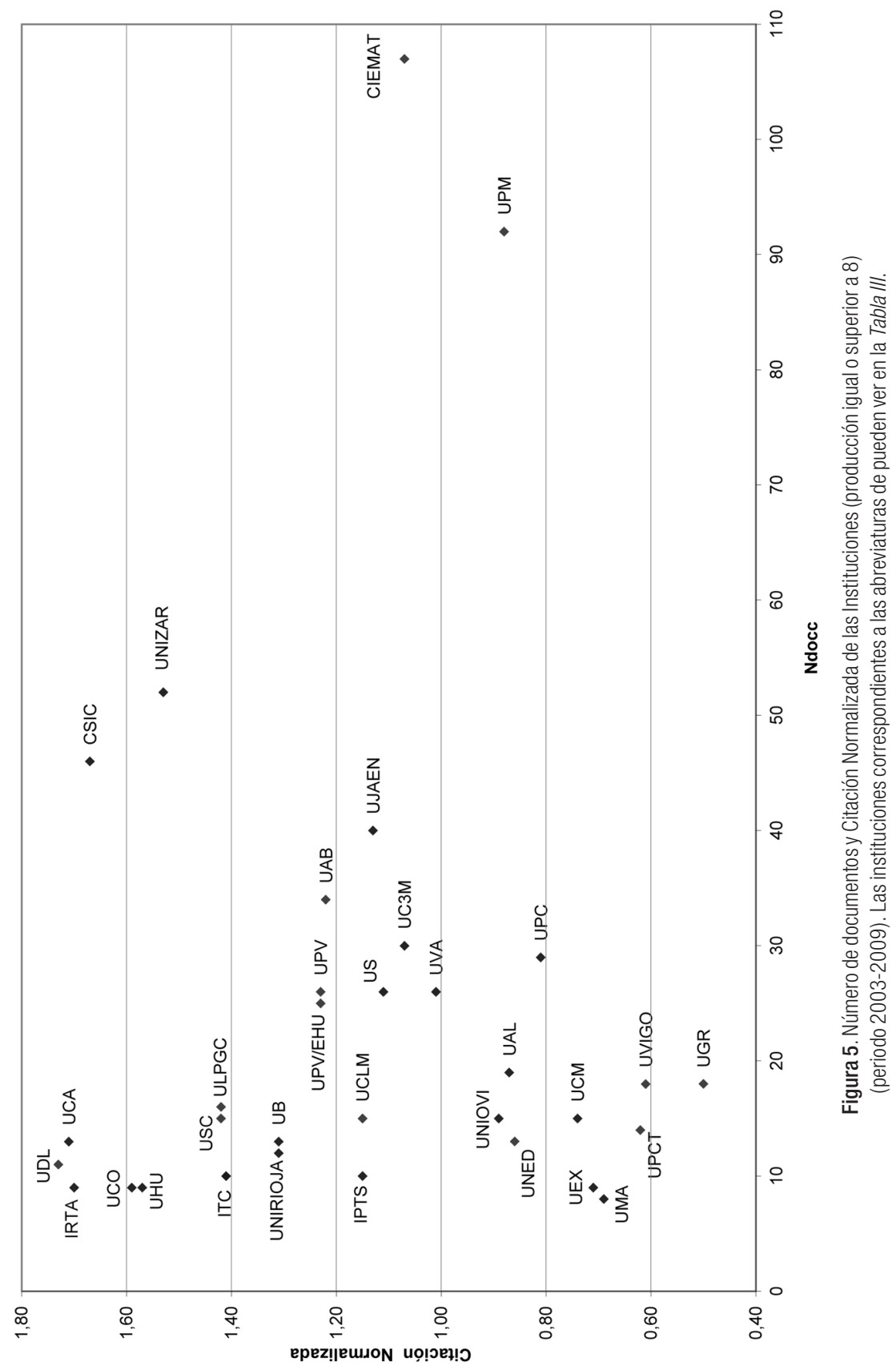


de la Universidad de Barcelona y de la Universidad de Santiago de Compostela, todas tuvieron una especialización superior al promedio mundial. El resto son la Universidad de La Rioja, la Universidad de las Palmas de Gran Canaria, la Universidad de Zaragoza, la Universidad de Huelva, la Universidad de Córdoba, la Universidad de Cádiz, la Universitat de Lleida y la Universitat Jaume I. Destacó la alta citación normalizada que obtuvo esta última con sus 8 trabajos.

- Grupo 3. Constituido por aquellas Universidades que obtuvieron una citación normalizada entre 1 y 1.3. Todas ellas tienen una especialización superior al promedio mundial y producciones medias; destaca la Universidad de Jaén con 9.55 y sus 40 trabajos. Además de la mencionada se encuentran la Universidad de Valladolid, la Universidad Carlos III de Madrid, la Universidad de Sevilla, la Universidad de Castilla-La Mancha, la Universitat Autónoma de Barcelona, la Universidad Politécnica de Valencia y la Universidad del País Vasco.

- Grupo 4. Caracterizado por incluir Universidades que obtuvieron una citación normalizada inferior al promedio mundial. La Universidad Complutense de Madrid fue la única con especialización inferior al promedio mundial. Destacó la producción y la especialización de la Universidad Politécnica de Madrid. El resto de universidades que integraban el grupo eran la Universidad de Málaga, la Universidad de Granada, la Universitat Politécnica de Catalunya, la Universidad de Oviedo, la Universidad de Extremadura, la Universidad de Vigo, la Universidad Nacional de Educación a Distancia, la Universidad Politécnica de Cartagena y la Universidad de Almería, y todas ellas tuvieron una producción media o baja.

La Tabla IV muestra cómo la revista Renewable Energy fue la que contó con mayor número de documentos citables españoles publicados, si bien en cuanto a citas descendió a la posición tercera. Seguida de ésta encontramos a Solar Energy, que en cuanto a citas descendió a la posición quinta.

Las revistas que mayor incremento en el número de documentos citables españoles experimentaron en el periodo estudiado fueron Renewable and Sustainable Energy Reviews, Energy Conversion and Management y Renewable Energy.

Las revistas que mayor número de citas obtienen en el periodo estudiado fueron Solar Energy Materials and Solar Cells y Energy Conversion and Management.

Annual Review of Environment and Resources y Solar Energy Materials and Solar Cells son las revistas que mayor impacto alcanzaron en el periodo, 
额

\begin{tabular}{|c|c|c|c|c|c|c|c|c|c|c|c|c|c|c|c|c|c|c|c|c|}
\hline $\begin{array}{l}\stackrel{?}{\mathcal{J}} \\
\gtrless\end{array}$ & $\frac{10}{\underset{\sigma}{\sigma}}$ & $\underset{\sim}{\stackrel{ \pm}{~}}$ & $\begin{array}{l}0 \\
\infty \\
\infty \\
\Gamma\end{array}$ & $\begin{array}{l}0 \\
0 \\
0 \\
0 \\
1\end{array}$ & $\mid \begin{array}{l}0 \\
0 \\
0 \\
0\end{array}$ & $\begin{array}{l}\tau \\
\infty \\
\infty\end{array}$ & $\begin{array}{l}\text { లె } \\
\text { ஓं }\end{array}$ & $\begin{array}{l}\infty \\
0 \\
\tilde{N} \\
\tilde{N}\end{array}$ & $\frac{}{\sigma}$ & $\begin{array}{l}\bar{\infty} \\
\underset{N}{N}\end{array}$ & $\stackrel{0}{\circ}$ & 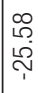 & & ○् & & 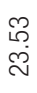 & & $\frac{\frac{L}{\sigma}}{\frac{\sigma}{1}}$ & & 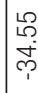 \\
\hline 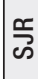 & \begin{tabular}{|l}
1 \\
0 \\
0 \\
0 \\
0
\end{tabular} & 용 & $\mid \begin{array}{l}\infty \\
0 \\
\vdots \\
0\end{array}$ & 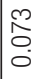 & $\mid \begin{array}{l}1 \\
0 \\
0 \\
0\end{array}$ & $\begin{array}{l}8 \\
0 \\
0\end{array}$ & $\begin{array}{l}\overline{0} \\
0 \\
0\end{array}$ & 告 & $\begin{array}{l}m \\
0 \\
0\end{array}$ & $\begin{array}{l}\overline{0} \\
0 \\
0\end{array}$ & 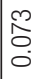 & $\begin{array}{l}0 \\
\text { O } \\
0 \\
0\end{array}$ & 占 & 둉 & \begin{tabular}{l}
1 \\
\multirow{2}{0}{} \\
0 \\
0
\end{tabular} & $\begin{array}{l}\text { L } \\
\text { L } \\
0 \\
0\end{array}$ & $\frac{1}{0}$ & $\begin{array}{l}\stackrel{\sim}{\circlearrowleft} \\
\stackrel{\circ}{\circ}\end{array}$ & $\begin{array}{l}\text { D } \\
0 \\
0 \\
0\end{array}$ & $\begin{array}{l}8 \\
8 \\
0 \\
0\end{array}$ \\
\hline & $\mid \begin{array}{l}\infty \\
\infty \\
c \\
\llcorner\end{array}$ & 预 & $\frac{N}{\tilde{N}}$ & হু & $\begin{array}{l}8 \\
80 \\
60 \\
10\end{array}$ & $\begin{array}{l}\stackrel{\sim}{\sim} \\
\underset{m}{m}\end{array}$ & $\begin{array}{l}\text { ㅇ } \\
\text { ஜ }\end{array}$ & 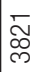 & $\stackrel{0}{\stackrel{N}{L}}$ & $\begin{array}{l}\infty \\
\infty \\
\sim \\
\sim\end{array}$ & $\begin{array}{l}\infty \\
\infty \\
\infty \\
\end{array}$ & $\stackrel{m}{\stackrel{m}{ }}$ & $\stackrel{\circ}{\stackrel{N}{\sim}}$ & $\underset{\mathbb{N}}{\stackrel{N}{N}}$ & $\frac{m}{\infty}$ & $\underset{⿱}{\frac{\infty}{\square}}$ & ঙ্లি & శ్లై & ז & $\underset{\sim}{\infty}$ \\
\hline
\end{tabular}

ชิ

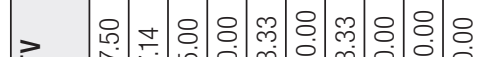

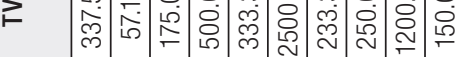

용

ฯ ตे

응 궁

$\gtrless$ 은

동

을 응을

के

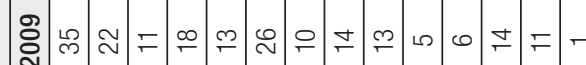

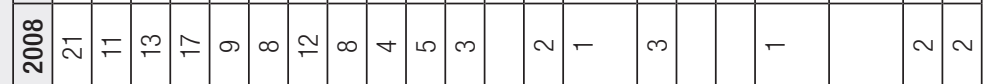

官

을 을

\&

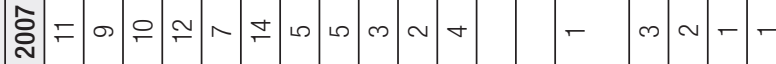

용.

突

ॠ ฮู

迸 高

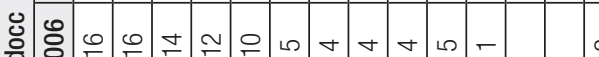

\begin{tabular}{|c|c|c|c|c|c|c|c|c|c|c|c|c|c|c|c|c|}
\hline ○్ & 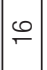 & $\dddot{0}$ & $\Xi$ & $\cong$ & 으 & مـ & $\nabla$ & $\nabla$ & $\nabla$ & 10 & - & $\sim$ & $\sim$ & - & م & $\nabla$ \\
\hline 용 & o & $\stackrel{\sim}{\sim}$ & 志 & $\sigma$ & $\infty$ & $\tau$ & $\sim$ & $\sim$ & & - & $\nabla$ & $m$ & $\nabla$ & $\sim$ & & $\sim$ \\
\hline
\end{tabular}

을

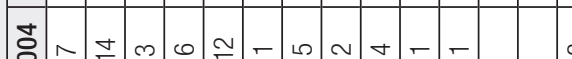

뜔 응

응 릉

오

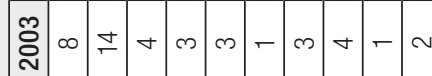

m $\sim \sim-$

官

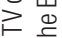

舫

응

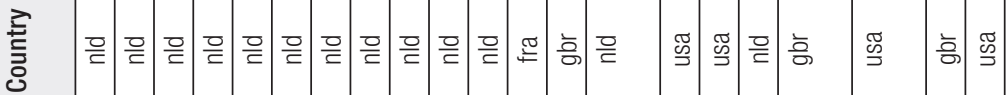

을

○ б б б б б б

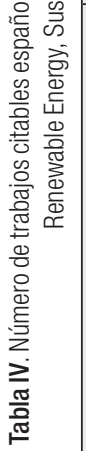

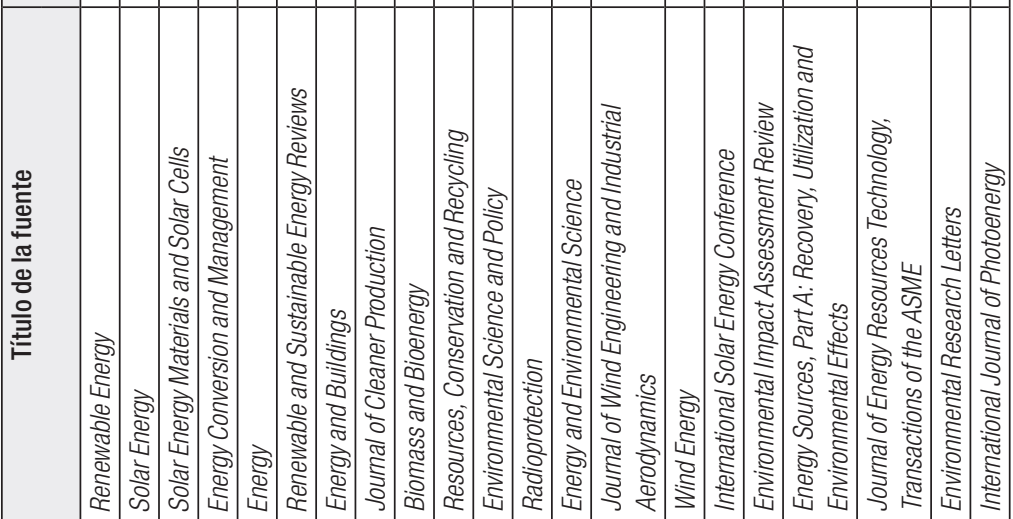




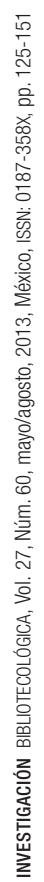

\begin{tabular}{|c|c|c|c|c|c|c|c|c|c|c|c|c|c|c|c|c|}
\hline & & $\begin{array}{l}\stackrel{0}{0} \\
\end{array}$ & & 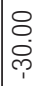 & & & & & & $\begin{array}{l}\pi \\
\mathbb{0} \\
8 \\
0\end{array}$ & & \begin{tabular}{l}
8 \\
8 \\
$\infty$ \\
\hdashline \\
0
\end{tabular} & & & & \\
\hline $\begin{array}{l}\stackrel{0}{0} \\
0 \\
0\end{array}$ & 足 & $\begin{array}{l}\stackrel{L}{\tilde{O}} \\
0 \\
0\end{array}$ & 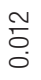 & $\begin{array}{l}0 \\
\tilde{o} \\
0 \\
0 \\
0\end{array}$ & $\hat{\sigma}$ & 号. & 茴 & $\frac{\infty}{\sigma}$ & Oे & 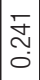 & $\begin{array}{l}\text { g. } \\
\text { Oे } \\
0\end{array}$ & 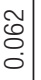 & 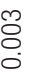 & . & & $\begin{array}{l}\text { Oे } \\
\text { ठ }\end{array}$ \\
\hline ని & $\simeq$ & $\stackrel{\sim}{N}$ & $\stackrel{\sim}{\sim}$ & $\bar{\sigma}$ & $\stackrel{2}{\sim}$ & $\begin{array}{l}\infty \\
\stackrel{\infty}{\leftarrow}\end{array}$ & 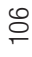 & ১ & ซ & ৪্নে & 㤩 & 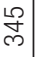 & & in & & 0 \\
\hline & & & & & & & & & & & & & & & 'ִ & \\
\hline ナ & $\nabla$ & m & $m$ & $m$ & $\sim$ & $\sim$ & $\sim$ & $\sim$ & $\sim$ & - & - & - & - & - & - & $\tau$ \\
\hline
\end{tabular}

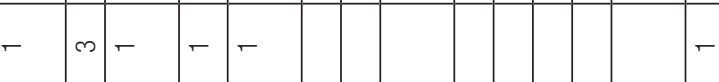

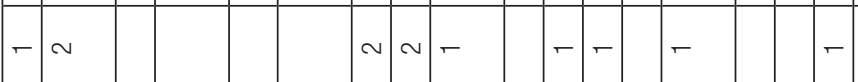

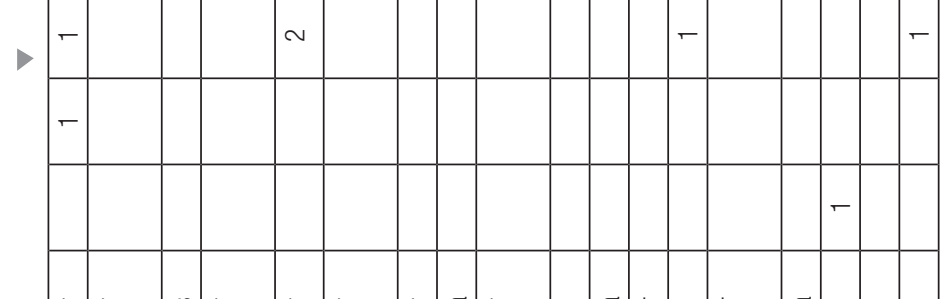

흥 흥 产흠 흥 흠

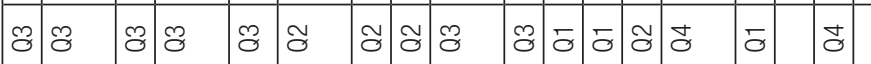

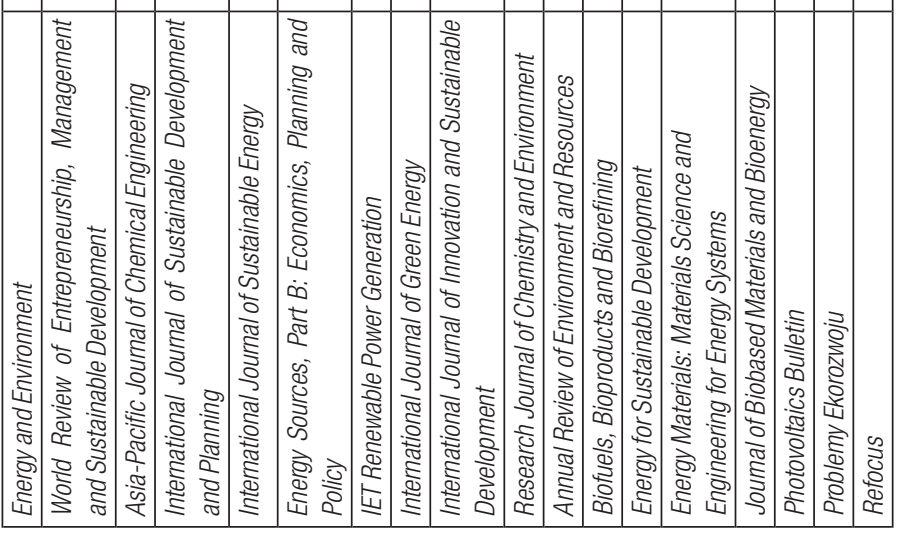


siendo esta última la que mayor crecimiento anual medio presentó para este indicador.

Tabla V. Evolución temporal del número de trabajos citables españoles en cada cuartil de las revistas de la categoría Renewable Energy, Sustainability and the Environment (periodo 2003-2009)

\begin{tabular}{|cccccc|}
\hline & ASSJR & $\begin{array}{c}\text { Q4 } \\
\text { (Lowest values) }\end{array}$ & Q3 & Q2 & $\begin{array}{c}\text { Q1 } \\
\text { (highest values) }\end{array}$ \\
2003 & 1.06 & 1 & & 15 & 31 \\
2004 & 1.08 & 2 & & 19 & 44 \\
2005 & 1.08 & 5 & & 21 & 71 \\
2006 & 1.05 & 2 & 9 & 16 & 82 \\
2007 & 1.06 & 2 & 5 & 19 & 71 \\
2008 & 1.03 & 6 & 3 & 34 & 90 \\
2009 & 1.04 & 2 & 7 & 94 & 103 \\
\hline
\end{tabular}

Como se puede ver en la Tabla $V$ el mayor número de trabajos de las revistas de la categoría Renewable Energy, Sustainability and the Environment se encuentran entre las revistas de mayor impacto, ya que se sitúan en el cuartil 1. A medida que pasaron los años se fueron incluyendo un mayor número de trabajos en las revistas del primer cuartil, excepto en el año 2007.

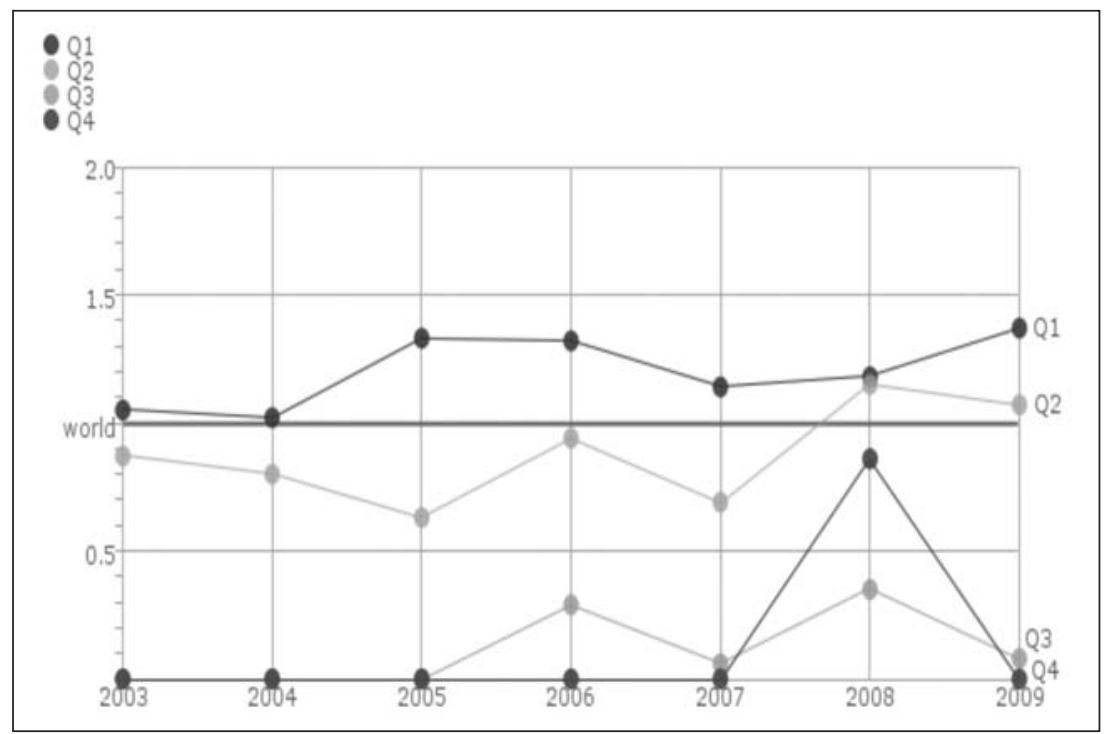

Figura 6. Evolución de la Citación Normalizada de los documentos citables españoles en el área de Renewable Energy, Sustainability and the Environment por cuartiles de las revistas de publicación 
Si analizamos los cuartiles de las revistas donde se publicaron los trabajos científicos citables españoles en el área de estudio y la citación normalizada, vemos cómo las del primer cuartil, que es donde más revistas encontramos, superaron al mundo.

Analizando los valores medios para algunas de las variables utilizadas, se puede llevar a cabo un estudio descriptivo de los grupos resultantes para las Revistas:

- Grupo 1. Caracterizado por tener un número alto de documentos, mayor número de citas en el periodo y mayor impacto. Todas las revistas de este grupo son holandesas y podemos decir que el prototipo de este grupo es Solar Energy Materials and Solar Cells. Otras revistas que incluimos en este grupo son: Energy Conversion and Management, Solar Energy y Biomass and Bioenergy. A nivel particular, Biomass and Bioenergy se aleja de los valores más altos en este estudio en cuanto a número de documentos a pesar de que el resto de indicadores la colocarían en este grupo.

- Grupo 2. Caracterizado por contar con un número de documentos medio por revista, altos valores en citas, pero el impacto alcanzado por las revistas fue bajo. Al igual que el conjunto anterior, todas las revistas eran holandesas y podemos decir que el prototipo de este grupo es Renewable Energy. Además, otras revistas incluidas en esta agrupación fueron: Energy, Energy and Buildings, Journal of Cleaner Production, Resources, Conservation and Recycling, Journal of Wind Engineering and Industrial Aerodynamics.

- Grupo 3. Caracterizado por incluir revistas con un número de documentos medio por revista pequeño, bajos valores en citas y escaso impacto. En este grupo predominan las revistas británicas y estadounidenses y es muy diferente al primer grupo. Podemos decir que el prototipo es Problemy Ekorozwoju, y además, incluimos las siguientes revistas en esta agrupación: Radioprotection, Energy and Environmental Science, Wind Energy, Environmental Impact Assessment Review, Energy Sources, Part A: Recovery, Utilization and Environmental Effects; Journal of Energy Resources Technology, Transactions of the ASME, International Journal of Photoenergy, Energy and Environment, World Review of Entrepreneurship, Management and Sustainable Development, International Journal of Sustainable Energy, Asia-Pacific Journal of Chemical Engineering, International Journal of Sustainable Development and Planning, International Journal of Innovation and Sustainable Development, Energy Sources, Part B: Economics, Planning 
and Policy; IET Renewable Power Generation, International Journal of Green Energy, Research Journal of Chemistry and Environment, Biofuels, Bioproducts and Biorefining y Journal of Biobased Materials and Bioenergy. A nivel individual Environmental Impact Assessment Review se aproxima a los promedios mundiales en cuanto a citas.

- Y por último, el grupo 4 se caracteriza por tener revistas con un número pequeño de documentos por revista, un número medio de citas, pero en este caso, con un mayor SJR. Podríamos decir que el prototipo sería Environmental Research Letters, además de la cual se incluyeron las siguientes revistas: Renewable and Sustainable Energy Reviews, Environmental Science and Policy, Annual Review of Environment and Resources y Energy for Sustainable Development. Tres de las revistas de este grupo son holandesas, una estadounidense y una británica. Renewable and Sustainable Energy Reviews obtuvo valores altos en cuanto a número de documentos y Annual Review of Environment and Resources fue la revista que obtuvo un impacto más alto no sólo en este grupo sino en el total de revistas analizadas.

\section{Conclusiones}

La producción científica en el área de estudio creció linealmente en el periodo 2003-2009 a nivel mundial, en España también creció linealmente pero más deprisa, y se cuadruplicó en el periodo. Durante el mismo la producción española ascendió desde la decimoquinta posición a la sexta. Esto significa que se incrementó mucho el esfuerzo investigador durante el periodo, a principios del cual, podríamos decir, estaba por debajo de su lugar natural. Este incremento de la producción no ha supuesto una merma de la calidad en la citación normalizada, sino que también se ha incrementado.

La tasa de colaboración en España en el área de estudio no es muy alta, de hecho los trabajos sin colaboración vienen a suponer cerca del $50 \%$. Se atisba una tendencia a decrecer. Igualmente se atisba una tendencia al crecimiento de la tasa de colaboración internacional, que viene a estar por encima del $25 \%$. Se comprueba que la colaboración internacional viene a suponer un incremento de la citación por regla general.

Con todo ello España viene a estar dentro del grupo de países sobresalientes, que tienen una gran producción científica con alta especialización temática reconocida internacionalmente.

Hay 109 instituciones españolas que publicaron trabajos en el área y periodo de estudio. De ellas solamente 34 tuvieron una producción promedio 
anual superior a un trabajo. Entre las mismas hubo 29 universidades y otras cinco instituciones del sector público que fueron clasificadas en cuatro grupos.

Las instituciones del sector público (grupo 1) son de las que mayor especialización tuvieron, salvo el CSIC que es más generalista. Destacaron el CIEMAT por su alta producción que supuso cerca del $15 \%$ de la producción nacional y el CSIC con una citación normalizada que superó en un 67 \% el promedio mundial.

Existen 10 universidades con una citación normalizada superior en un $30 \%$ al promedio mundial (grupo 2), entre las que destacó la Universidad de Zaragoza que sin producción en 2003 se colocó en primera posición en 2009 junto con el CIEMAT. Por debajo del promedio mundial hay 11 universidades (grupo 4), entre las que destacó la UPM por su producción y especialización. De las restantes 8 universidades (grupo 3 ) que se encontraban en una situación intermedia destacó la U. de Jaén por su producción y especialización.

Y por último, la mayoría de las revistas utilizadas para publicar por los científicos españoles del área de estudio fueron del primer cuartil, seguidas de las del segundo a gran distancia de las revistas del tercer y cuarto cuartil. Esto explica los buenos resultados de impacto obtenidos. Gran parte de ellas figuraron como holandesas por pertenecer al grupo Elsevier, el mayor grupo editorial de revistas científicas. En concreto, las dos revistas más utilizadas a gran distancia del resto fueron Renewable Energy y Solar Energy.

Esto también da una idea de la fortaleza del impulso de las energías renovables y en concreto de la solar dentro del área de estudio.

De todo ello se puede inferir que ha habido un incremento del esfuerzo científico dedicado al área de estudio durante el periodo estudiado, sin que dicho esfuerzo pueda atribuirse exclusivamente a nuevas instituciones o científicos, sino más bien a un cambio de orientación de las mismas. Esto hizo posible no sólo el aumento de la producción sino también del impacto, lo que mejoró las posiciones de España en el ranking mundial. Esto puso de manifiesto que el esfuerzo inversor llevado a cabo a nivel europeo, con el VII programa marco, apoyado a nivel nacional con el VI Plan Nacional de Investigación Científica, Desarrollo e Innovación Tecnológica, obtuvo un gran resultado en España. No así en el resto de Europa que pese a haber incrementado su producción perdió porcentaje en la producción mundial porque en otras partes del mundo el incremento fue mayor.

\section{Agradecimientos}

Este trabajo fue financiado por el Plan Nacional de Investigación Cientifi- 
Desarrollo Regional (FEDER) como parte de los proyectos de investigación TIN2008-06514-C02-01 y TIN2008-06514-C02-02.

\section{BibliografíA}

Archambault, É.; Campbell, D.; Gingras, Y.; Larivière, V. (2009), “Comparing Bibliometric Statistics Obtained from the Web of Science and Scopus", en Journal of the American Society for Information Science and Technology (JASIST), 60 (7), 1320-1326.

Bollen, J.; Van de Sompel, H.; Hagberg, A.; Chute, R. (2009), “A principal component analysis of 39 scientific impact measures", en PLOS ONE, 4(6), e6022 DOI:10.1371/journal,pone,0006022.

Brundtland, G. et al. (1987), Our Common Future: Brundtland Report, disponible en: http://worldinbalance.net/intagreements/1987-brundtland. php [consultado el 9 de octubre de 2012].

Celiktas, M. S.; Sevgili, T.; Kocar, G. (2009), “A snapshot of renewable energy research in Turkey", en Renewable Energy, 34, 1479-1486.

Codina, L. (2005), "Scopus: el mayor navegador científico de la web", en El Profesional de la Información, 4, 44-49.

Dong, B.; Xu, G.; Luo, X.; Cai, Y.; Gao, W. (2012), "A bibliometric analysis of solar power research from 1991 to 2010”, en Scientometrics, 93, 11011117.

González-Pereira, B.; Guerrero-Bote, V. P.; Moya-Anegón, F. (2010), “A new approach to the metric of journals' scientific prestige: The SJR indicator", en Journal of Informetrics, 4, 379-391.

Guerrero-Bote, V. P.; Moya-Anegón, F. (2012), "A further step forward in measuring journals' scientific prestige: The SJR2 indicator”, en Journal of Informetrics, 6, 674-688.

Guerrero-Bote, V. P.; Olmeda-Gómez, C.; Moya-Anegón, F. (2013), "Quantifying the Benefits of International Scientific Collaboration”, en Journal of the American Society for Information Science and Technology (en prensa), DOI: 10.1002 /asi.22754.

Hane, P. (2004), "Elsevier announces Scopus service", en Information today, disponible en: http://newsbreaks.infotoday.com/nbreader.asp?Ar ticleID=16494 [consulta: 11 diciembre 2011]

Hassan, E. (2005), “The evolution of the knowledge structure of fuel cells", en Scientometrics, 62 (2), 223-238.

Jacso, P. (2004), “Scopus, Péter's Digital Reference Shelf”, en Gale Cengage Learning, disponible en: http://www.gale.cengage.com/servlet/HTML FileServlet imprint $=9999 \&$ region $=7 \&$ fileName $=/$ reference/archive $/$ 200409/scopus.html [consulta: 15 diciembre 2011].

Kajikawa, Y.; Takeda, Y. (2008), "Structure of research on biomass and bio-fuels: A citation-based approach", en Technological Forecasting and Social Change, 75 (9), 1349-1359. 
Kajikawa, Y.; Yoshikawa, J.; Takeda, Y.; Matsushima, K. (2008), "Tracking emerging technologies in energy research: Toward a roadmap for sustainable energy", en Technological Forecasting and Social Change, 75, 771-782.

Komiyama, H.; Takeuchi, K. (2006), "Sustainability science: building a new discipline”, en Sustainability Science, 1, 1-6.

Laguardia, C. (2005), "E-views and reviews: Scopus vs. Web of Science", en Library Journal, 15, disponible en: http://www.library journal.com/article/CA491154.html [consulta: 3 enero 2012].

Lancho-Barrantes, B.; Guerrero-Bote, V. P.; Chinchilla-Rodríguez, Z.; Moya-Anegón, F. (2012), "Citation flows in the zones of influence of scientific collaborations", en Journal of the American Society for Information Science and Technology, 63 (3), 481-489.

Leydesdorff, L.; Moya Anegón, F.; Guerrero Bote, V. P. (2010), “Journal maps on the basis of Scopus data: A comparison with the Journal Citation Reports of the ISI", en Journal of the American Society for Information Science and Technology, 61(2), 352-369.

Moya-Anegón, F.; Chinchilla Rodríguez, Z.; Vargas Quesada, B.; Corera Álvarez, E.; Muñoz Fernández, F. J.; González Molina, A.; Herrero Solana, V. (2007), "Coverage analysis of Scopus: A journal metric approach”, en Scientometrics, 73(1), 53-78.

Moya-Anegón, F.; Chinchilla Rodríguez, Z.; Corera Álvarez, E.; Muñoz Fernández, F.; Vargas Quesada, B.; Herrero Solana, V. (2004), Indicadores Bibliométricos de la Actividad Científica Española: ISI, Web of Science, 1998-2002, Madrid: FECYT.

Romo-Fernández, L. M.; Guerrero-Bote, V. P.; Moya-Anegón, F. (2012), "World scientific production on renewable energy, sustainability and the environment", en Energy for Sustainable Development, 16, 500-508, DOI:10.1016/j.esd.2012.06.005

Romo-Fernández, L. M.; López-Pujalte, C.; Guerrero-Bote, V. P.; Moya-Anegón, F. (2011), "Analysis of Europe's scientific production on renewable energies", en Renewable Energy, 36(9), 2529. 2537.

Sanz-Casado, E.; García-Zorita, J. C.; Serrano-López, A. E.; Larsen, B.; Ingwersen, P. (2012), "Renewable energy research 1995-2009: a case study of wind power research in EU, Spain, Germany and Denmark", en Scientometrics (in press), Scimago Institutions Rankings, disponible en http://www.scimagoir.com/methodology.php [consulta: 20 diciembre 2012].

Schubert, A.; Lang, I. (2005), "The Literature Aftermath Of The Brundtland Report 'Our Common Future'. A Scientometric Study Based On Citations In Science And Social Science Journals", en Environment, Development and Sustainability, 7, 1-8, DOI: 10.1007/s10668-003-0177-5

Simon, C.; Batchman, T.; Taylor, C. (2010), "Introduction to renewable energy: An interdisciplinary approach", en ASEE Annual Conference and Exposition, Conference Proceedings, $16 \mathrm{pp}$. 
Thomas, S. M. (1992), "The evaluation of plant biomass research: a case study of the problems inherent in bibliometric indicators", en Scientometrics, 23(1), 149-167.

Tsay, M. (2008), "A bibliometric analysis of hydrogen energy literature, 1965-2005”, en Scientometrics, 75(3), 421-438.

Uzun, A. (2002), "National patterns of research output and priorities in renewable energy", en Energy Policy, 30, 131-136. 